Journal of Mathematics and Statistics 7 (3): 207-215, 2011

ISSN 1549-3644

(C) 2011 Science Publications

\title{
Flows of Continuous-Time Dynamical Systems with No Periodic Orbit as an Equivalence Class under Topological Conjugacy Relation
}

\author{
Tahir Ahmad and Tan Lit Ken \\ Department of Mathematics, Faculty of Science, Nanotechnology Research Alliance, \\ Theoretical and Computational Modeling for Complex Systems, \\ University Technology Malaysia 81310 Skudai, Johor, Malaysia
}

\begin{abstract}
Problem statement: Flows of continuous-time dynamical systems with the same number of equilibrium points and trajectories, and which has no periodic orbit form an equivalence class under the topological conjugacy relation. Approach: Arbitrarily, two trajectories resulting from two distinct flows of this type of dynamical systems were written as a set of points (orbit). A homeomorphism which maps between these two sets is then built. Using the notion of topological conjugacy, they were shown to conjugate topologically. By the arbitrariness in selection of flows and their respective initial states, the results were extended to all the flows of dynamical system of that type. Results: Any two flows of such dynamical systems were shown to share the same dynamics temporally along with other properties such as order isomorphic and homeomorphic. Conclusion: Topological conjugacy serves as an equivalence relation in the set of flows of continuous-time dynamical systems which have same number of equilibrium points and trajectories, and has no periodic orbit.
\end{abstract}

Key words: Dynamical system, equilibrium points, trajectories, periodic orbit, equivalence class, topological conjugacy, order isomorphic, Flat Electroencephalography (Flat EEG), dynamical systems

\section{INTRODUCTION}

Dynamical system is a system where its temporal evolution from some initial state is dictated by a set of rules (Eduard et al., 1999). Another way to understand this is, it consists of a set of variables that describe its state and a law that describe the evolution of the state variables with time (i.e., how the state of the system in the next moment of time depends on the input and its state in the previous moment of time) (Eugene, 2007). There exist various classifications of dynamical systems, e.g. continuous-time (flow or semiflow) or discrete-time, continuous state or discrete state and linear or non linear. In order to study the system of interest more richly, some authors require the state space to possess certain specific structure such as compact Hausdorff space as in (Tim and Justin, 1989), separable metric space (Flytzanis, 1976), or even smooth manifold as define in (Artur, 1979). For some survey on the definition of dynamical system we direct the readers to (Chen, 2000; Ling and Anthony, 2007; Sekhar et al., 1999).

Dynamical systems have been used in many areas of research, e.g. fluid flow analysis, economic processes (stock market models), physics, medicine, meteorology, astronomy, and population growth models. Nandhakumar et al. (2009) for example, the dynamics of robot arm is studied. On the other hand, (Krishan et al., 2010) shows that by adopting neurofuzzy system, the design of robust controllers for uncertain non-linear dynamical systems can be done without resorting to system model simplifications and linearization and without imposing structural conditions on the system uncertainties. This shows that the use of concept of dynamical system is vast. Mark and Marc (2005), the importance of dynamical systems as an approach in understanding development was discussed. One power of dynamical systems approach is that we can tell something or many things, about a system without knowing all the details that govern the system evolution (Eugene, 2007). With the use of this mathematical theory, it allows one to talk about stability, equilibrium, bifurcations.

This study is motivated by the study in (Tahir and Tan, 2010), where the authors shows that the dynamics of an epilepsy patients who is having seizure, modeled as a continuous dynamical system can be transported to Flat Electroencephalography (Flat EEG) that are modeled in the same way. This study basically generalizes the theorems obtained from (Tahir and Tan, 2010).

Corresponding Author: Tahir Ahmad, Department of Mathematics, Faculty of Science, Nanotechnology Research Alliance, Theoretical and Computational Modeling for Complex Systems,

University Technology Malaysia 81310 Skudai, Johor, Malaysia 
Literature review: Some dynamical systems are governed by differential equations and this is the type of dynamical systems that will be discussed in this study. A system of differential equations is relations between its functions and its derivatives (James, 2007). Sometimes they are referred to as vector field because they assign a vector (direction and magnitude) to each point in the state space (also known as phase space). Two general classes of system of differential equations are autonomous and non-autonomous. Systems that depend explicitly on time are referred to as non-autonomous or time-dependent vector fields and those which do not depend explicitly on time are referred to as autonomous. Every non-autonomous vector fields can be made into autonomous by redefining the time as a new dependent variable. Thus, for simplification purposes we will just speak of continuous-time dynamical system from this point onwards.

The type of dynamical system that we will be discussing in this study will be of continuous-time dynamical system non-periodic orbit where the evolution rule is a flow. An example usage on the flow concept in different area of research is in (Adeqbie and Alao, 2007), where the temperature-dependent viscous fluid between parallel heated walls is modeled as a flow. According to (James, 2007), a flow $\phi_{t}(\mathrm{x})$ is a oneparameter differentiable mapping $\phi: \Re \times X \rightarrow X$, such that it fulfills two properties, which are:

$\phi_{0}(\mathrm{x})=\mathrm{x} \quad \forall \mathrm{x} \in \mathrm{X}$

And for all $t$ and $S \in \mathfrak{R}$ :

$\phi_{\mathrm{t}} \circ \phi_{\mathrm{s}}=\phi_{\mathrm{t}+\mathrm{s}}$

where the composition symbol, ${ }^{\circ}$ means $\phi_{\mathrm{t}}{ }^{\circ} \phi_{\mathrm{s}}(\mathrm{x})=$ $\phi_{\mathrm{t}}\left(\phi_{\mathrm{s}}(\mathrm{x})\right)$.

For each $\mathrm{x} \in \mathrm{X}, \phi_{\mathrm{t}}(\mathrm{x})$ defines a curve in $\mathrm{X}$ as $\mathrm{t}$ varies over $\Re$. This curve is known as orbit or trajectory. A consequence from the property (b) which is also known as group property is that two distinct trajectories will not cross. A property of flow is that it is differentiable, therefore there is an associated ordinary differential equations or more precisely a vector field which assigns a vector (magnitude and direction) to each of the points in the state space (James, 2007). To visualize this, consider a point chosen from the state space, this point will generate a curve in the state space. The movement of this point from one state to another is according to the evolution rule (flow) and the tangent vector to each point on this curve is exactly the vectors computed by using the vector field:

$$
f(x)=\frac{d}{d t} \phi_{t}(x) \quad \ni t=0
$$

Suppose $\left\{\phi_{\mathrm{t}}\left(\mathrm{x}_{0}\right): t \in \Re\right\}$ is an orbit starting at $\mathrm{x}_{0} \in \mathrm{X}$, then this orbit is periodic if for each $x \in\left\{\phi_{t}\left(x_{0}\right): t \in \Re\right\}$, there exist a time $T \in \Re$ such that $\phi_{\mathrm{t}}(\mathrm{x})=\mathrm{x}$. In our discussion, we restrict our dynamical system to those that has no periodic orbit (since it is not possible to construct a homeomorphism from a loop to $\mathfrak{R}$ ), those with the same number of equilibrium points (since a single point cannot be homeomorphic to $\mathfrak{R}$ ) and with the same dimension in terms of their state space. We also assume that the number of trajectories of the systems discussed to be same so that our homeomorphism will be constructible. For some survey on periodic system, readers may read (Baryarama et al., 2005) where the periodicity of the HIV/AIDS epidemic in a mathematical model that incorporates complacency is discussed or (Ibrahim et al., 2007) for the periodic and non periodic (complex) behavior of a model of bioreactor with cell recycling.

\section{MATERIALS AND METHODS}

Let $\mathrm{x}_{0} \in \mathrm{X}$, be a initial state such that $\phi_{0}\left(\mathrm{x}_{0}\right)=\mathrm{x}_{0}$, then the flow will traces out an orbit or trajectory. Denote this trajectory as $\mathrm{O}_{\phi_{\mathrm{t}}\left(\mathrm{x}_{\mathrm{o}}\right)}=\left\{\phi_{\mathrm{t}}\left(\mathrm{x}_{\mathrm{o}}\right): \mathrm{t} \in \mathfrak{R}\right\}$. Note that the set $\Re$ is linearly ordered by the usual less than or equal relation, $\leq$. Taking account of this property, it is not difficult to see that elements in the set $\mathrm{O}_{\phi_{t}\left(x_{0}\right)}$ is also linearly ordered, with the ordering relation be defined as follows

$$
\left(\mathrm{x}_{\mathrm{i}}, \mathrm{t}_{\mathrm{i}}\right) \prec_{\hat{\phi}_{\mathrm{t}}\left(\mathrm{x}_{\mathrm{o}}\right)}\left(\mathrm{x}_{\mathrm{j}}, \mathrm{t}_{\mathrm{j}}\right) \Leftrightarrow \mathrm{t}_{\mathrm{i}} \leq \mathrm{t}_{\mathrm{j}}
$$

Therefore, the pair $\left(\mathrm{O}_{\phi_{1}\left(x_{0}\right)}, \prec_{\phi_{1}\left(x_{0}\right)}\right)$ is linearly ordered and we have lemma 1. As an example, see (Tahir et al., 2005), where the state space trajectory of seizure is augmented and shown to exhibit linear ordering properties. The construction is however a little different from the one we have just presented above because the formulation of dynamical system they started off from is slightly different. Nevertheless, they actually meant the same as the augmented trajectory is simply the evolution of states in seizure over an interval of time.

Lemma 1: $\left(O_{\phi_{t}\left(x_{0}\right)}, \prec_{\phi_{t}\left(x_{0}\right)}\right)$ is linearly ordered.

Now, according to (Steve, 2008), every linear ordering, $\leq$ can induce a strict linear ordering, $<$ which can be written as:

$\mathrm{x}<\mathrm{y}$ if $\mathrm{x} \leq \mathrm{y}$ and $\mathrm{x} \neq \mathrm{y}$ 
Thus, we can certainly "transform" the linearly ordered set $\left(\mathrm{O}_{\phi t(\mathrm{xo})}, \prec_{\phi t(\mathrm{xo})}\right)$ into a strictly linearly ordered set $\left(\mathrm{O}_{\phi \mathrm{t}(\mathrm{xo})}, \prec{ }_{\phi t}(\mathrm{xo})\right)$, with the ordering relation defined as:

$\left(\mathrm{x}_{\mathrm{i}}, \mathrm{t}_{\mathrm{i}}\right) \prec_{\phi_{\mathrm{t}}\left(\mathrm{x}_{\mathrm{o}}\right)}\left(\mathrm{x}_{\mathrm{j}}, \mathrm{t}_{\mathrm{j}}\right)$

If:

$$
\left(\mathrm{x}_{\mathrm{i}}, \mathrm{t}_{\mathrm{i}}\right) \prec_{\dot{\phi}_{\mathrm{t}}\left(\mathrm{x}_{\mathrm{o}}\right)}^{\cdot}\left(\mathrm{x}_{\mathrm{j}}, \mathrm{t}_{\mathrm{j}}\right)
$$

And:

$$
\left(\mathrm{x}_{\mathrm{i}}, \mathrm{t}_{\mathrm{i}}\right) \neq\left(\mathrm{x}_{\mathrm{j}}, \mathrm{t}_{\mathrm{j}}\right)
$$

Or simply:

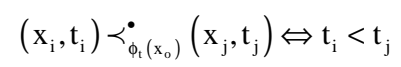

And this can be stated formally as.

Lemma 2: $\left(\mathrm{O}_{\phi_{\mathrm{t}}\left(\mathrm{x}_{\mathrm{o}}\right)}, \prec_{\phi_{\mathrm{t}}\left(\mathrm{x}_{\mathrm{o}}\right)}\right)$ is strictly linearly ordered.

Therefore, we have formulated a strictly linearly ordered set from a particular trajectory which resulted from a flow of an arbitrary continuous-time dynamical system. Before we proceed further, let us emphasize once that, whenever we speak continuous-time dynamical system from this point onwards, we meant those with the properties stated earlier. In the following, we explain how the trajectory can be composed into a topological space.

Using the definition of interval topology in (Kopperman et al., 1998) one can define a topology on a strictly linearly ordered set by using a subbasis which consists of the collection of all order open rays that can be defined as follow:

$$
\mathrm{S}_{\mathrm{O}_{\phi_{\mathrm{t}}\left(\mathrm{x}_{\mathrm{o}}\right)}}=\left\{\begin{array}{l}
\left\{(\mathrm{x}, \mathrm{t}) \in \mathrm{O}_{\phi_{\mathrm{t}}\left(\mathrm{x}_{\mathrm{o}}\right)} \mid(\mathrm{x}, \mathrm{t}) \prec_{\dot{\phi}_{\mathrm{t}}\left(\mathrm{x}_{\mathrm{o}}\right)}^{\cdot}(\mathrm{a}, \mathrm{t})\right\} \\
,\left\{(\mathrm{x}, \mathrm{t}) \in \mathrm{O}_{\phi_{\mathrm{t}}\left(\mathrm{x}_{\mathrm{o}}\right)} \mid(\mathrm{b}, \mathrm{t}){\dot{\dot{\phi}_{\mathrm{t}}\left(\mathrm{x}_{\mathrm{o}}\right)}}^{\cdot}(\mathrm{x}, \mathrm{t})\right\} \\
, \varphi, \mathrm{O}_{\phi_{\mathrm{t}}\left(\mathrm{x}_{\mathrm{o}}\right)}: \forall(\mathrm{a}, \mathrm{t}),(\mathrm{b}, \mathrm{t}) \in \mathrm{O}_{\phi_{\mathrm{t}}\left(\mathrm{x}_{\mathrm{o}}\right)}
\end{array}\right\}
$$

Equation 1 will generate a topology called interval topology. The elements of the topology are the union of any finite intersections of elements of Eq. 1. Note that each element in Eq. 1 is itself open in the topology generated by it. We denote this topology as:

$$
\tau_{\sigma_{q_{l}\left(x_{0}\right)}}=\left\{\alpha \mid \alpha=\bigcup_{i=1}\left(\bigcap_{j=1}^{n} s_{j}\right)_{i}: s_{j} \in S_{O_{q_{l}\left(x_{0}\right)}}\right\}
$$

Consequently, the trajectory is composed into a topological space $\left(\mathrm{O}_{\phi \mathrm{t}(\mathrm{xo})}, \tau_{\mathrm{o \phi t}(\mathrm{xo})}\right)$, namely linearly ordered topological space (LOTS). LOTS are also a Generalized Ordered space (GO-space) (Bennet and Lutzer, 1996). In fact, the class of GO-spaces is exactly the class of all subspaces of LOTS (Bennet et al., 2001). Hence from (Kopperman et al., 1998), which says that a GO-space is Hausdorff, we have $\left(\mathrm{O}_{\phi_{t}\left(\mathrm{x}_{\mathrm{o}}\right)}, \tau_{\mathrm{O}_{\phi_{\mathrm{t}}\left(\mathrm{x}_{0}\right)}}\right)$ as a Hausdorff LOTS.

Lemma 3: $\left(\mathrm{O}_{\phi(\mathrm{xo})}, \tau_{\mathrm{o \phi t}(\mathrm{xo})}\right)$ is a Hausdorff LOTS.

Now consider two distinct flows from two continuous-time dynamical systems with no periodic orbit, $\phi: \Re \times X \rightarrow X$ and $\psi: \Re \times Y \rightarrow Y$ such that the dimension of the state space of $X$ and $Y$ are same. Using the above argument, we then have two strictly linearly ordered set $\left(\mathrm{O}_{\phi_{\mathrm{t}}\left(\mathrm{x}_{\mathrm{o}}\right)}, \prec_{\dot{\phi}_{\mathrm{t}}\left(\mathrm{x}_{\mathrm{o}}\right)}\right)$ and $\left(\mathrm{O}_{\psi_{\mathrm{t}}\left(\mathrm{y}_{\mathrm{o}}\right)}, \prec_{\psi_{\mathrm{t}}\left(\mathrm{y}_{\mathrm{o}}\right)}\right)$ and this two strictly linearly ordered set can be composed into LOTS (Table 1).

From Table 1 we notice the existence of the following lemma.

Lemma 4: Let $\left(\mathrm{x}_{\mathrm{i}}, \mathrm{t}_{\mathrm{i}}\right)$ represents a point on a trajectory $\mathrm{O}_{\phi_{\mathrm{t}}\left(\mathrm{x}_{\mathrm{o}}\right)}$ at time $\mathrm{t}_{\mathrm{i}}$. Similarly, let $\left(\mathrm{y}_{\mathrm{i}}, \mathrm{t}_{\mathrm{i}}\right)$ represents another point on another trajectory $\mathrm{O}_{\psi_{\mathrm{t}}\left(\mathrm{y}_{\mathrm{o}}\right)}$ at time $\mathrm{t}_{\mathrm{i}}$. Then, for $\mathrm{t}_{\mathrm{i}} \leq \mathrm{t}_{\mathrm{j}}$, a point $\left(\mathrm{x}_{\mathrm{i}}, \mathrm{t}_{\mathrm{i}}\right)$ on the trajectory $\mathrm{O}_{\phi_{\mathrm{t}}\left(\mathrm{x}_{\mathrm{o}}\right)}$ precede another point $\left(\mathrm{x}_{\mathrm{j}}, \mathrm{t}_{\mathrm{j}}\right)$ if and only if a point $\left(\mathrm{y}_{\mathrm{i}}, \mathrm{t}_{\mathrm{i}}\right)$ on another trajectory $\mathrm{O}_{\psi_{1}\left(y_{\mathrm{o}}\right)}$ precede another point $\left(\mathrm{y}_{\mathrm{j}}, \mathrm{t}_{\mathrm{j}}\right)$. In other words:

$$
\left(\mathrm{x}_{1}, \mathrm{t}_{1}\right) \prec_{\phi_{\mathrm{t}}\left(\mathrm{x}_{\mathrm{o}}\right)}\left(\mathrm{x}_{2}, \mathrm{t}_{2}\right) \Leftrightarrow\left(\mathrm{y}_{1}, \mathrm{t}_{1}\right) \prec_{\psi_{1}\left(\mathrm{y}_{\mathrm{o}}\right)}\left(\mathrm{y}_{2}, \mathrm{t}_{2}\right)
$$

Proof: From the linear order of continuous-time dynamical system 1 (Table 1) we have:

$$
\left(\mathrm{x}_{\mathrm{i}}, \mathrm{t}_{\mathrm{i}}\right) \prec_{\phi_{\mathrm{t}}\left(\mathrm{x}_{\mathrm{o}}\right)}\left(\mathrm{x}_{\mathrm{j}}, \mathrm{t}_{\mathrm{j}}\right) \Leftrightarrow \mathrm{t}_{\mathrm{i}} \leq \mathrm{t}_{\mathrm{j}}
$$

And from the linear order of continuous-time dynamical system 2 (Table 1) we have:

$$
\left(\mathrm{y}_{\mathrm{i}}, \mathrm{t}_{\mathrm{i}}\right) \prec_{\psi_{\mathrm{t}}\left(\mathrm{y}_{\mathrm{o}}\right)}\left(\mathrm{y}_{\mathrm{j}}, \mathrm{t}_{\mathrm{j}}\right) \Leftrightarrow \mathrm{t}_{\mathrm{i}} \leq \mathrm{t}_{\mathrm{j}}
$$

Combining these two, we will have:

$$
\left(\mathrm{x}_{1}, \mathrm{t}_{1}\right) \prec_{\phi_{\mathrm{t}}\left(\mathrm{x}_{\mathrm{o}}\right)}\left(\mathrm{x}_{2}, \mathrm{t}_{2}\right) \Leftrightarrow\left(\mathrm{y}_{1}, \mathrm{t}_{1}\right) \prec_{\psi_{\mathrm{t}}\left(\mathrm{y}_{\mathrm{o}}\right)}\left(\mathrm{y}_{2}, \mathrm{t}_{2}\right)
$$

As desired.

Next, define a function $\theta: \mathrm{O}_{\phi_{t}\left(x_{\mathrm{o}}\right)} \rightarrow \mathrm{O}_{\psi_{\mathrm{t}}\left(\mathrm{y}_{\mathrm{o}}\right)}$ as

$$
\theta((\mathrm{x}, \mathrm{t}))=(\mathrm{y}, \mathrm{t})
$$


J. Math. \& Stat., 7 (3): 207-215, 2011

Table 1: Two continuous-time dynamical system

\begin{tabular}{|c|c|c|}
\hline Continuous-time dynamical system 1 & Type & Continuous-time dynamical system 2 \\
\hline$\phi: \Re \times X \rightarrow X \quad \ni X=\Re^{n}$ & Flow & $\psi: \Re \times Y \rightarrow Y \quad \ni \mathrm{Y}=\Re^{\mathrm{n}}$ \\
\hline $\mathrm{O}_{\phi_{\mathrm{t}}\left(\mathrm{x}_{\mathrm{o}}\right)}=\left\{\phi_{\mathrm{t}}\left(\mathrm{x}_{\mathrm{o}}\right): \mathrm{t} \in \mathfrak{R}\right\}$ & Orbit & $\mathrm{O}_{\psi_{\mathrm{t}}\left(\mathrm{y}_{\mathrm{o}}\right)}=\left\{\psi_{\mathrm{t}}\left(\mathrm{y}_{\mathrm{o}}\right): \mathrm{t} \in \mathfrak{R}\right\}$ \\
\hline$\left(\mathrm{x}_{\mathrm{i}}, \mathrm{t}_{\mathrm{i}}\right) \prec_{\phi_{\mathrm{t}}\left(\mathrm{x}_{\mathrm{o}}\right)}\left(\mathrm{x}_{\mathrm{j}}, \mathrm{t}_{\mathrm{j}}\right) \Leftrightarrow \mathrm{t}_{\mathrm{i}} \leq \mathrm{t}_{\mathrm{j}}$ & Linear order relation & $\left(\mathrm{y}_{\mathrm{i}}, \mathrm{t}_{\mathrm{i}}\right) \prec_{\psi_{\mathrm{t}}\left(\mathrm{y}_{\mathrm{o}}\right)}\left(\mathrm{y}_{\mathrm{j}}, \mathrm{t}_{\mathrm{j}}\right) \Leftrightarrow \mathrm{t}_{\mathrm{i}} \leq \mathrm{t}_{\mathrm{j}}$ \\
\hline$\left(\mathrm{O}_{\phi_{\mathrm{t}}\left(\mathrm{x}_{\mathrm{o}}\right)}, \prec_{\phi_{\mathrm{t}}\left(\mathrm{x}_{\mathrm{o}}\right)}\right)$ & Linearly ordered set & $\left(\mathrm{O}_{\psi_{\mathrm{t}}\left(\mathrm{y}_{\mathrm{o}}\right)}, \prec_{\psi_{\mathrm{t}}\left(\mathrm{y}_{\mathrm{o}}\right)}\right)$ \\
\hline$\left(\mathrm{x}_{\mathrm{i}}, \mathrm{t}_{\mathrm{i}}\right) \prec_{\phi_{\mathrm{t}}\left(\mathrm{x}_{\mathrm{o}}\right)}^{\cdot}\left(\mathrm{x}_{\mathrm{j}}, \mathrm{t}_{\mathrm{j}}\right) \Leftrightarrow \mathrm{t}_{\mathrm{i}}<\mathrm{t}_{\mathrm{j}}$ & Strict linear order & $\left(\mathrm{y}_{\mathrm{i}}, \mathrm{t}_{\mathrm{i}}\right) \prec_{\psi_{\mathrm{t}}\left(\mathrm{y}_{\mathrm{o}}\right)}^{\cdot}\left(\mathrm{y}_{\mathrm{j}}, \mathrm{t}_{\mathrm{j}}\right) \Leftrightarrow \mathrm{t}_{\mathrm{i}}<\mathrm{t}_{\mathrm{j}}$ \\
\hline$\left(\mathrm{O}_{\phi_{1}\left(\mathrm{x}_{\mathrm{o}}\right)}, \prec_{\phi_{\mathrm{t}}\left(\mathrm{x}_{\mathrm{o}}\right)}^{\cdot}\right)$ & Strictly linearly order & $\left(\mathrm{O}_{\psi_{\mathrm{t}}\left(\mathrm{y}_{\mathrm{o}}\right)}, \prec_{\psi_{\mathrm{t}}\left(\mathrm{y}_{\mathrm{o}}\right)}\right)$ \\
\hline $\mathrm{S}_{\mathrm{O}_{\phi_{\mathrm{t}}\left(\mathrm{x}_{\mathrm{o}}\right)}}=\left\{\begin{array}{l}\left\{(\mathrm{x}, \mathrm{t}) \in \mathrm{O}_{\phi_{\mathrm{t}}\left(\mathrm{x}_{\mathrm{o}}\right)} \mid(\mathrm{x}, \mathrm{t}) \prec_{\phi_{\mathrm{t}}\left(\mathrm{x}_{\mathrm{o}}\right)}(\mathrm{a}, \mathrm{t})\right\}, \\
\left\{(\mathrm{x}, \mathrm{t}) \in \mathrm{O}_{\phi_{\mathrm{t}}\left(\mathrm{x}_{\mathrm{o}}\right)} \mid(\mathrm{b}, \mathrm{t}) \prec_{\phi_{\mathrm{t}}\left(\mathrm{x}_{\mathrm{o}}\right)}(\mathrm{x}, \mathrm{t})\right\}, \\
\varphi, \mathrm{O}_{\phi_{\mathrm{t}}\left(\mathrm{x}_{\mathrm{o}}\right)} \\
: \forall(\mathrm{a}, \mathrm{t}),(\mathrm{b}, \mathrm{t}) \in \mathrm{O}_{\phi_{\mathrm{t}}\left(\mathrm{x}_{\mathrm{o}}\right)}\end{array}\right\}$ & Subbasis & $\mathrm{S}_{\mathrm{O}_{\psi_{1}\left(y_{0}\right)}}=\left\{\begin{array}{l}\left\{(\mathrm{y}, \mathrm{t}) \in \mathrm{O}_{\psi_{\mathrm{t}}\left(\mathrm{y}_{\mathrm{o}}\right)} \mid(\mathrm{y}, \mathrm{t}) \prec_{\psi_{\mathrm{t}}\left(\mathrm{y}_{\mathrm{o}}\right)}^{\cdot}(\mathrm{c}, \mathrm{t})\right\}, \\
\left\{(\mathrm{y}, \mathrm{t}) \in \mathrm{O}_{\psi_{\mathrm{t}}\left(\mathrm{y}_{\mathrm{o}}\right)} \mid(\mathrm{d}, \mathrm{t}) \prec_{\psi_{\mathrm{t}}\left(\mathrm{y}_{\mathrm{o}}\right)}^{\cdot}(\mathrm{y}, \mathrm{t})\right\}, \\
\varphi, \mathrm{O}_{\psi_{\mathrm{t}}\left(\mathrm{y}_{\mathrm{o}}\right)} \\
: \forall(\mathrm{c}, \mathrm{t}),(\mathrm{d}, \mathrm{t}) \in \mathrm{O}_{\psi_{\mathrm{t}}\left(\mathrm{y}_{\mathrm{o}}\right)}\end{array}\right.$ \\
\hline$\tau_{\mathrm{O}_{\varphi_{1}\left(x_{0}\right)}}=\left\{\alpha \mid \alpha=\bigcup_{\mathrm{i}=1}\left(\bigcap_{\mathrm{j}=1} \mathrm{~s}_{\mathrm{j}}\right)_{\mathrm{i}}: \mathrm{s}_{\mathrm{j}} \in \mathrm{S}_{\mathrm{O}_{\phi_{1}\left(\mathrm{x}_{0}\right)}}\right\}$ & Interval topology & $\tau_{\mathrm{O}_{\psi_{1}\left(y_{0}\right)}}=\left\{\beta \mid \beta=\bigcup_{\mathrm{i}=1}\left(\bigcap_{j=1} \mathrm{~s}_{\mathrm{j}}\right)_{\mathrm{i}}: \mathrm{s}_{\mathrm{j}} \in \mathrm{S}_{\mathrm{O}_{\psi_{\mathrm{t}}\left(\mathrm{y}_{\mathrm{o}}\right)}}\right\}$ \\
\hline$\left(\mathrm{O}_{\phi_{\mathrm{t}}\left(\mathrm{x}_{\mathrm{o}}\right)}, \tau_{\mathrm{O}_{\phi_{\mathrm{t}}\left(\mathrm{x}_{\mathrm{o}}\right)}}\right)$ & Hausdorff LOTS & $\left(\mathrm{O}_{\psi_{\mathrm{t}}\left(\mathrm{y}_{\mathrm{o}}\right)}, \tau_{\mathrm{O}_{\psi_{1}\left(y_{\mathrm{o}}\right)}}\right)$ \\
\hline
\end{tabular}

Theorem 1: $\theta((\mathrm{x}, \mathrm{t}))=(\mathrm{y}, \mathrm{t})$ is a bijective function.

\section{Proof:}

Function: Suppose $\left(\mathrm{x}_{\mathrm{i}}, \mathrm{t}_{\mathrm{i}}\right)=\left(\mathrm{x}_{\mathrm{j}}, \mathrm{t}_{\mathrm{j}}\right)$

Then by lemma $4,\left(y_{i}, t_{i}\right)=\left(y_{j}, t_{j}\right)$

or $\theta\left(\left(\mathrm{x}_{\mathrm{i}}, \mathrm{t}_{\mathrm{i}}\right)\right)=\theta\left(\left(\mathrm{x}_{\mathrm{j}}, \mathrm{t}_{\mathrm{j}}\right)\right)$

Thus, $\theta((\mathrm{x}, \mathrm{t}))=(\mathrm{y}, \mathrm{t})$ is indeed a function.

Injective: Suppose $\theta\left(\left(\mathrm{x}_{\mathrm{i}}, \mathrm{t}_{\mathrm{i}}\right)\right)=\theta\left(\left(\mathrm{x}_{\mathrm{j}}, \mathrm{t}_{\mathrm{j}}\right)\right)$

By the defined function, this implies:

$\left(\mathrm{y}_{\mathrm{i}}, \mathrm{t}_{\mathrm{i}}\right)=\left(\mathrm{y}_{\mathrm{j}}, \mathrm{t}_{\mathrm{j}}\right)$

Now by lemma 4, we then have:

$\left(x_{i}, t_{i}\right)=\left(x_{j}, t_{j}\right)$

Thus, $\theta((x, t))=(y, t)$ is injective.

Surjective: For all $(\mathrm{y}, \mathrm{t}) \in \mathrm{O}_{\psi_{\mathrm{t}}\left(\mathrm{y}_{\mathrm{o}}\right)}$
There exist $(\mathrm{x}, \mathrm{t}) \in \mathrm{O}_{\phi_{\mathrm{t}}\left(\mathrm{x}_{\mathrm{o}}\right)}$

Such that $\theta((x, t))=(y, t)$

Therefore, $\theta((x, t))=(y, t)$ is surjective

Since $\theta((x, t))=(y, t)$ is injective and surjective, thus it is bijective.

Next, using the bijective function we show that $\left(\mathrm{O}_{\phi_{1}\left(x_{0}\right)}, \prec_{\phi_{1}\left(x_{0}\right)}\right)$ and $\left(\mathrm{O}_{\psi_{1}\left(y_{0}\right)}, \prec_{\psi_{t}\left(y_{0}\right)}\right)$ are order isomorphic.

Theorem 2: $\left(\mathrm{O}_{\phi_{\mathrm{t}}\left(\mathrm{x}_{\mathrm{o}}\right)}, \prec_{\phi_{\mathrm{t}}\left(\mathrm{x}_{\mathrm{o}}\right)}\right)$ is order isomorphic to $\left(\mathrm{O}_{\psi_{\mathrm{t}}\left(\mathrm{y}_{\mathrm{o}}\right)}, \prec_{\psi_{\mathrm{t}}\left(\mathrm{y}_{\mathrm{o}}\right)}\right)$

Proof: From lemma 4 we have:

$\left(\mathrm{x}_{1}, \mathrm{t}_{1}\right) \prec_{\phi_{\mathrm{t}}\left(\mathrm{x}_{\mathrm{o}}\right)}\left(\mathrm{x}_{2}, \mathrm{t}_{2}\right) \Leftrightarrow\left(\mathrm{y}_{1}, \mathrm{t}_{1}\right) \prec_{\psi_{\mathrm{t}}\left(\mathrm{y}_{\mathrm{o}}\right)}\left(\mathrm{y}_{2}, \mathrm{t}_{2}\right)$

Substituting the function $\theta((x, t))=(y, t)$ into this lemma, we then have

$\left(\mathrm{x}_{1}, \mathrm{t}_{1}\right) \prec_{\phi_{1}\left(\mathrm{x}_{\mathrm{o}}\right)}\left(\mathrm{x}_{2}, \mathrm{t}_{2}\right) \Leftrightarrow \theta\left(\mathrm{x}_{1}, \mathrm{t}_{1}\right) \prec_{\psi_{1}\left(\mathrm{y}_{\mathrm{o}}\right)} \theta\left(\mathrm{x}_{2}, \mathrm{t}_{2}\right)$

Therefore, $\left(\mathrm{O}_{\phi_{1}\left(\mathrm{x}_{\mathrm{o}}\right)}, \prec_{\phi_{\mathrm{t}}\left(\mathrm{x}_{\mathrm{o}}\right)}\right)$ is order isomorphic to $\left(\mathrm{O}_{\psi_{\mathrm{t}}\left(\mathrm{y}_{\mathrm{o}}\right)}, \prec_{\psi_{\mathrm{t}}\left(\mathrm{y}_{\mathrm{o}}\right)}\right)$. 
We now proceed to show that $\theta((\mathrm{x}, \mathrm{t}))=(\mathrm{y}, \mathrm{t})$ is a homeomorphism.

Theorem 3: The function $\theta((x, t))=(y, t)$ is continuous.

Proof: Consider the set

$$
\mathrm{S}_{\mathrm{O}_{\psi_{1}\left(y_{\mathrm{o}}\right)}}=\left\{\begin{array}{l}
\left\{(\mathrm{y}, \mathrm{t}) \in \mathrm{O}_{\psi_{\mathrm{t}}\left(\mathrm{y}_{\mathrm{o}}\right)} \mid(\mathrm{y}, \mathrm{t}) \prec_{\psi_{\mathrm{t}}\left(\mathrm{y}_{\mathrm{o}}\right)}^{\cdot}(\mathrm{c}, \mathrm{t})\right\} \\
,\left\{(\mathrm{y}, \mathrm{t}) \in \mathrm{O}_{\psi_{\mathrm{t}}\left(\mathrm{y}_{\mathrm{o}}\right)} \mid(\mathrm{d}, \mathrm{t}) \bullet_{\psi_{\mathrm{t}}\left(\mathrm{y}_{\mathrm{o}}\right)}(\mathrm{y}, \mathrm{t})\right\} \\
, \varphi, \mathrm{O}_{\psi_{\mathrm{t}}\left(\mathrm{y}_{\mathrm{o}}\right)}: \forall(\mathrm{a}, \mathrm{t}),(\mathrm{b}, \mathrm{t}) \in \mathrm{O}_{\psi_{\mathrm{t}}\left(\mathrm{y}_{\mathrm{o}}\right)}
\end{array}\right\}
$$

Case 1: Sets of the form:

$$
\left\{(\mathrm{y}, \mathrm{t}) \in \mathrm{O}_{\psi_{\mathrm{t}}\left(\mathrm{y}_{\mathrm{o}}\right)} \mid(\mathrm{y}, \mathrm{t}) \prec_{\psi_{\mathrm{t}}\left(\mathrm{y}_{\mathrm{o}}\right)}(\mathrm{c}, \mathrm{t})\right\}
$$

Clearly:

$$
\begin{aligned}
& \theta^{-1}\left(\left\{(\mathrm{y}, \mathrm{t}) \in \mathrm{O}_{\psi_{\mathrm{t}}\left(\mathrm{y}_{\mathrm{o}}\right)} \mid(\mathrm{y}, \mathrm{t}) \prec_{\dot{\psi}_{\mathrm{t}}\left(\mathrm{y}_{\mathrm{o}}\right)}(\mathrm{c}, \mathrm{t})\right\}\right) \\
& =\left\{(\mathrm{x}, \mathrm{t}) \in \mathrm{O}_{\phi_{\mathrm{t}}\left(\mathrm{x}_{\mathrm{o}}\right)} \mid(\mathrm{x}, \mathrm{t}) \dot{\bullet}_{\dot{\phi}_{\mathrm{t}}\left(\mathrm{x}_{\mathrm{o}}\right)}(\mathrm{a}, \mathrm{t})\right\}
\end{aligned}
$$

For some $(a, t) \in O_{\phi_{t}\left(x_{0}\right)}$, since $\theta^{-1}$ is bijective.

Notice that the set $\left\{(\mathrm{x}, \mathrm{t}) \in \mathrm{O}_{\dot{\phi}_{1}\left(\mathrm{x}_{\mathrm{o}}\right)} \mid(\mathrm{x}, \mathrm{t}) \prec_{\dot{\phi}_{\mathrm{t}}\left(\mathrm{x}_{\mathrm{o}}\right)}^{\cdot}(\mathrm{a}, \mathrm{t})\right\}$ is an order-open ray in $O_{\phi_{t}\left(x_{0}\right)}$, i.e. element of subbasis. Since $\left\{(\mathrm{x}, \mathrm{t}) \in \mathrm{O}_{\phi_{\mathrm{t}}\left(\mathrm{x}_{\mathrm{o}}\right)} \mid(\mathrm{x}, \mathrm{t}) \prec_{\phi_{\mathrm{t}}\left(\mathrm{x}_{\mathrm{o}}\right)}^{\cdot}(\mathrm{a}, \mathrm{t})\right\}$ is an order-open ray Thus, $\left\{(\mathrm{x}, \mathrm{t}) \in \mathrm{O}_{\phi_{\mathrm{t}}\left(\mathrm{x}_{\mathrm{o}}\right)} \mid(\mathrm{x}, \mathrm{t}){\dot{\phi_{\mathrm{t}}\left(\mathrm{x}_{\mathrm{o}}\right)}}^{\cdot}(\mathrm{a}, \mathrm{t})\right\}$ is an open set in $\mathrm{O}_{\phi_{t}\left(x_{0}\right)}$.

Case 2: Sets of the form:

$$
\left\{(\mathrm{y}, \mathrm{t}) \in \mathrm{O}_{\psi_{\mathrm{t}}\left(\mathrm{y}_{\mathrm{o}}\right)} \mid(\mathrm{d}, \mathrm{t}) \prec_{\psi_{\mathrm{t}}\left(\mathrm{y}_{\mathrm{o}}\right)}^{\cdot}(\mathrm{y}, \mathrm{t})\right\}
$$

Clearly:

$$
\begin{aligned}
& \theta^{-1}\left(\left\{(\mathrm{y}, \mathrm{t}) \in \mathrm{O}_{\psi_{\mathrm{t}}\left(\mathrm{y}_{\mathrm{o}}\right)} \mid(\mathrm{d}, \mathrm{t}) \prec_{\psi_{\mathrm{t}}\left(\mathrm{y}_{\mathrm{o}}\right)}^{\cdot}(\mathrm{y}, \mathrm{t})\right\}\right) \\
& =\left\{(\mathrm{x}, \mathrm{t}) \in \mathrm{O}_{\phi_{\phi_{1}\left(\mathrm{x}_{\mathrm{o}}\right)}} \mid(\mathrm{b}, \mathrm{t}) \prec_{\dot{\phi}_{\mathrm{t}}\left(\mathrm{x}_{\mathrm{o}}\right)}(\mathrm{x}, \mathrm{t})\right\}
\end{aligned}
$$

For some $(b, t) \in \mathrm{O}_{\phi_{t}\left(x_{0}\right)}$, since $\theta^{-1}$ is bijective.

Notice that the set $\left\{(\mathrm{x}, \mathrm{t}) \in \mathrm{O}_{\phi_{\mathrm{t}}\left(\mathrm{x}_{\mathrm{o}}\right)} \mid(\mathrm{b}, \mathrm{t}){\dot{\phi_{\mathrm{t}}\left(\mathrm{x}_{\mathrm{o}}\right)}}(\mathrm{x}, \mathrm{t})\right\}$ is an order-open ray in $O_{\phi_{1}\left(x_{0}\right)}$, i.e., element of subbasis.
Since $\left\{(\mathrm{x}, \mathrm{t}) \in \mathrm{O}_{\phi_{\mathrm{t}}\left(\mathrm{x}_{\mathrm{o}}\right)} \mid(\mathrm{b}, \mathrm{t}) \prec_{\dot{\phi}_{\mathrm{t}}\left(\mathrm{x}_{\mathrm{o}}\right)}^{\cdot}(\mathrm{x}, \mathrm{t})\right\}$ is an order-open ray.

Thus, $\left\{(\mathrm{x}, \mathrm{t}) \in \mathrm{O}_{\dot{\phi}_{\mathrm{t}}\left(\mathrm{x}_{\mathrm{o}}\right)} \mid(\mathrm{b}, \mathrm{t}) \prec_{\dot{\phi}_{\mathrm{t}}\left(\mathrm{x}_{\mathrm{o}}\right)}(\mathrm{x}, \mathrm{t})\right\}$ is an open set in $\mathrm{O}_{\phi_{t}\left(\mathrm{x}_{\mathrm{o}}\right)}$.

Case 3: The set $\phi$, i.e., empty set.

Clearly, $\theta^{-1}(\phi)=\phi$ (trivial). Since $\varphi \in \mathrm{S}_{\mathrm{O}_{\rho_{l}\left(x_{0}\right)}}$

Thus, $\phi$ is an open set in $\mathrm{O}_{\phi_{t}\left(x_{0}\right)}$.

Case 4: The set $O_{\psi_{1}\left(y_{0}\right)}$, i.e. the whole set.

Clearly, $\theta^{-1}\left(\mathrm{O}_{\psi_{\mathrm{t}}\left(\mathrm{y}_{\mathrm{o}}\right)}\right)=\mathrm{O}_{\phi_{\mathrm{t}}\left(\mathrm{x}_{\mathrm{o}}\right)}$ since $\theta^{-1}$ is surjective.

Since $\mathrm{O}_{\phi_{\mathrm{t}}\left(\mathrm{x}_{\mathrm{o}}\right)} \in \mathrm{S}_{\mathrm{O}_{\mathrm{e}_{1}\left(\mathrm{x}_{0}\right)}}$ Thus, $\mathrm{O}_{\phi_{\mathrm{t}}\left(\mathrm{x}_{\mathrm{o}}\right)}$ is an open set in $\mathrm{O}_{\phi_{1}\left(\mathrm{x}_{\mathrm{o}}\right)}$.

Combining these four cases, we have the inverse image of each element in the subbasis $S_{\mathrm{O}_{y_{1}\left(y_{0}\right)}}$ is open in $\left(\mathrm{O}_{\phi_{\mathrm{t}}\left(\mathrm{x}_{\mathrm{o}}\right)}, \tau_{\mathrm{O}_{\mathrm{t}_{\mathrm{t}}\left(\mathrm{x}_{0}\right)}}\right)$. Thus, $\theta((\mathrm{x}, \mathrm{t}))=(\mathrm{y}, \mathrm{t})$ is a continuous function that maps from $\left(\mathrm{O}_{\phi_{\mathrm{t}}\left(\mathrm{x}_{\mathrm{o}}\right)}, \tau_{\mathrm{O}_{\hat{\varphi}_{1}\left(x_{0}\right)}}\right)$ to $\left(\mathrm{O}_{\psi_{\mathrm{t}}\left(\mathrm{y}_{\mathrm{o}}\right)}, \tau_{\mathrm{O}_{\psi_{1}\left(y_{\mathrm{o}}\right)}}\right)$.

Theorem 4: The function $\theta^{-1}((y, t))=(x, t)$ is continuous .

Proof: Use similar argument in the proof for theorem 3. In that case, the function $\theta^{-1}((y, t))=(x, t)$ is bijective, continuous and open. Thus, it is a homeomorphism from $\left(\mathrm{O}_{\phi_{1}\left(x_{0}\right)}, \tau_{O_{\phi_{1}\left(x_{0}\right)}}\right)$ to $\left(O_{\psi_{1}\left(y_{0}\right)}, \tau_{O_{\psi_{1}\left(y_{0}\right)}}\right)$. Next we show that the flows of the two continuous-time dynamical system are topologically conjugated temporally. The topological notion of conjugacy used will be the one introduced in (James, 2007). This topological notion of conjugacy is one of the usual ways to relate two dynamical systems (Erik and Joseph, 2010). In fact, it is one of the most useful and interesting among the different possibilities of introducing an equivalence relation to classify dynamical systems (Nguyen, 1996). To achieve this, we conjugate two trajectories resulting from two different flows, and then by the fact that the initial state chosen is arbitrary, this result were extended to the rest pair of trajectories in the state spaces. Start off by defining two functions that acts on two different set of points (trajectories) which results from two different flows, respectively as:

$$
\phi_{\mathrm{x}_{0}}: \mathrm{O}_{\phi_{\mathrm{t}}\left(\mathrm{x}_{\mathrm{o}}\right)} \rightarrow \mathrm{O}_{\phi_{\mathrm{t}}\left(\mathrm{x}_{\mathrm{o}}\right)} \text { and } \psi_{\mathrm{y}_{0}}: \mathrm{O}_{\psi_{\mathrm{t}}\left(\mathrm{y}_{\mathrm{o}}\right)} \rightarrow \mathrm{O}_{\psi_{\mathrm{t}}\left(\mathrm{y}_{\mathrm{o}}\right)}
$$


Where:

$\phi_{\mathrm{x}_{0}}\left(\mathrm{x}_{\mathrm{i}}, \mathrm{t}_{\mathrm{i}}\right)=\left(\mathrm{x}_{\mathrm{i}+1}, \mathrm{t}_{\mathrm{i}+1}\right) \quad \forall\left(\mathrm{x}_{\mathrm{i}}, \mathrm{t}_{\mathrm{i}}\right) \in \mathrm{O}_{\phi_{\mathrm{t}}\left(\mathrm{x}_{\mathrm{o}}\right)}$ and $\mathrm{t}_{\mathrm{i}} \in \mathfrak{R}$

And:

$\psi_{\mathrm{y}_{0}}\left(\mathrm{y}_{\mathrm{i}}, \mathrm{t}_{\mathrm{i}}\right)=\left(\mathrm{y}_{\mathrm{i}+1}, \mathrm{t}_{\mathrm{i}+1}\right) \quad \forall\left(\mathrm{y}_{\mathrm{i}}, \mathrm{t}_{\mathrm{i}}\right) \in \mathrm{O}_{\psi_{\mathrm{t}}\left(\mathrm{y}_{\mathrm{o}}\right)}$ and $\mathrm{t}_{\mathrm{i}} \in \Re$

Then it will be these two functions which we will show they are topologically conjugated.

Theorem 5: $\quad \phi_{x_{0}}: \mathrm{O}_{\phi_{\mathrm{t}}\left(\mathrm{x}_{\mathrm{o}}\right)} \rightarrow \mathrm{O}_{\phi_{\mathrm{t}}\left(\mathrm{x}_{\mathrm{o}}\right)} \quad$ and $\psi_{y_{0}}: O_{\psi_{t}\left(y_{o}\right)} \rightarrow \mathrm{O}_{\psi_{t}\left(y_{o}\right)}$ is topologically conjugated.

Proof: For any $\left(\mathrm{x}_{\mathrm{i}-1}, \mathrm{t}_{\mathrm{i}-1}\right) \in \mathrm{O}_{\phi_{\mathrm{t}}\left(\mathrm{x}_{\mathrm{o}}\right)}$

Composition of functions $\theta$ and $\phi_{x_{0}}$ is

$\theta\left[\phi_{\mathrm{x}_{0}}\left(\mathrm{x}_{\mathrm{i}-1}, \mathrm{t}_{\mathrm{i}-1}\right)\right]$

$=\theta\left(x_{i}, t_{i}\right)$

$=\left(\mathrm{y}_{\mathrm{i}}, \mathrm{t}_{\mathrm{i}}\right)$

Composition of functions $\psi_{y_{0}}$ and $\theta$ is

$\psi_{\mathrm{y}_{0}}\left[\theta\left(\mathrm{x}_{\mathrm{i}-1}, \mathrm{t}_{\mathrm{i}-1}\right)\right]$

$=\psi_{\mathrm{y}_{0}}\left(\mathrm{y}_{\mathrm{i}-1}, \mathrm{t}_{\mathrm{i}-1}\right)$

$=\left(\mathrm{y}_{\mathrm{i}}, \mathrm{t}_{\mathrm{i}}\right)$

This shows that $\theta \circ \phi_{\mathrm{x}_{0}}=\psi_{\mathrm{y}_{0}} \circ \theta$

Thus, the two trajectories are topologically conjugated.

In constructing this proof, the point chosen as an initial state in both trajectories is arbitrary, as such for the rest of pair of points, their trajectories can also be conjugated topologically using similar method presented above. In that case, the two flows, $\phi: \Re \times X \rightarrow X$ and $\psi: \Re \times Y \rightarrow Y$ can be conjugated topologically by the homeomorphism $\mathrm{h}: \mathrm{X} \times \mathfrak{R} \rightarrow \mathrm{Y} \times \mathfrak{R}$, which can be defined as:

$$
\mathrm{h}(\mathrm{x}, \mathrm{t})=\left\{\begin{array}{l}
\theta_{0}(\mathrm{x}, \mathrm{t}) \text { if }(\mathrm{x}, \mathrm{t}) \in \mathrm{O}_{\phi_{\mathrm{t}}\left(\mathrm{x}_{\mathrm{o}}\right)} \text { where } \\
\theta_{0}: \mathrm{O}_{\phi_{\mathrm{t}}\left(\mathrm{x}_{\mathrm{o}}\right)} \rightarrow \mathrm{O}_{\psi_{\mathrm{t}}\left(\mathrm{y}_{\mathrm{o}}\right)} \text { and } \theta_{0} \circ \phi_{\mathrm{x}_{0}}=\psi_{\mathrm{y}_{0}} \circ \theta_{0} \\
\theta_{1}(\mathrm{x}, \mathrm{t}) \text { if }(\mathrm{x}, \mathrm{t}) \in \mathrm{O}_{\phi_{\mathrm{t}}\left(\mathrm{x}_{\mathrm{t}}\right)} \\
\text { where } \theta_{1}: \mathrm{O}_{\phi_{\mathrm{t}}\left(\mathrm{x}_{\mathrm{t}}\right)} \rightarrow \mathrm{O}_{\psi_{\mathrm{t}}\left(\mathrm{y}_{\mathrm{y}}\right)} \text { and } \theta_{1} \circ \phi_{\mathrm{x}_{1}}=\psi_{\mathrm{y}_{1}} \circ \theta_{1} \\
\theta_{2}(\mathrm{x}, \mathrm{t}) \text { if }(\mathrm{x}, \mathrm{t}) \in \mathrm{O}_{\phi_{\mathrm{t}}\left(\mathrm{x}_{2}\right)} \\
\text { where } \theta_{2}: \mathrm{O}_{\phi_{\mathrm{t}}\left(\mathrm{x}_{2}\right)} \rightarrow \mathrm{O}_{\psi_{\mathrm{t}}\left(\mathrm{y}_{2}\right)} \text { and } \theta_{2} \circ \phi_{\mathrm{x}_{2}}=\psi_{\mathrm{y}_{2}} \circ \theta_{2} \ldots \\
\theta_{\mathrm{m}}(\mathrm{x}, \mathrm{t}) \text { if }(\mathrm{x}, \mathrm{t}) \in \mathrm{O}_{\phi_{\mathrm{t}}\left(\mathrm{x}_{\mathrm{m}}\right)} \\
\text { where } \theta_{\mathrm{m}}: \mathrm{O}_{\phi_{\mathrm{t}}\left(\mathrm{x}_{\mathrm{m}}\right)} \rightarrow \mathrm{O}_{\psi_{\mathrm{t}}\left(\mathrm{y}_{\mathrm{m}}\right)} \text { and } \theta_{\mathrm{m}} \circ \phi_{\mathrm{x}_{\mathrm{m}}}=\psi_{\mathrm{y}_{\mathrm{m}}} \circ \theta_{\mathrm{m}}
\end{array}\right.
$$

For all $(\mathrm{x}, \mathrm{t}) \in \mathrm{X} \times \mathfrak{R},(\mathrm{y}, \mathrm{t}) \in \mathrm{Y} \times \mathfrak{R}$, and $\mathrm{m} \in \mathrm{N}$.

Notice that our claim that the homeomorphism, $h$ defined above conjugate the two flows topologically must be true. This is clear from the properties of flow given earlier where two distinct trajectories will not intersect and that the property (a) for flow is defined for all $x \in X$. Thus, we can imagine the state space of the dynamical system as a space filled with a set of trajectories. In fact, the union of all these trajectories form the state space set. For example, if the state space of a flow is the Euclidean space $\mathfrak{R}^{2}$, then for each point on the state space, there will be a trajectory, and the union of all this trajectories will be the set $\mathfrak{R}^{2}$. This can also be visualized as the flow partitions the state space into classes where each class represents a trajectory and our method in conjugating the two flows is by conjugating each pair of these trajectories. As the two flows were chosen arbitrary, thus we conclude all the above results in the following theorems.

Theorem 6: Any two continuous-time dynamical systems with no periodic orbit are:

- Order-isomorphic

- Homeomorphic

- Topologically conjugated temporally

Proof: Let $\phi: \Re \times X \rightarrow X$ and $\psi: \Re \times Y \rightarrow Y$ be two flows of continuous-time dynamical systems with nonperiodic orbit. For all $\left(\mathrm{x}_{\mathrm{i}-1}, \mathrm{t}_{\mathrm{i}-1}\right) \in \mathrm{X} \times \mathfrak{R}$ and $\mathrm{h}: \mathrm{X} \times \Re \rightarrow \mathrm{Y} \times \Re$ be defined as (2):

$\mathrm{h} \circ \phi\left(\mathrm{x}_{\mathrm{i}-1}, \mathrm{t}_{\mathrm{i}-1}\right)$

$=\mathrm{h}\left(\mathrm{x}_{\mathrm{i}}, \mathrm{t}_{\mathrm{i}}\right)$

$=\left(\mathrm{y}_{\mathrm{i}}, \mathrm{t}_{\mathrm{i}}\right)$

$\psi \circ \mathrm{h}\left(\mathrm{x}_{\mathrm{i}-1}, \mathrm{t}_{\mathrm{i}-1}\right)$

$=\psi\left(\mathrm{y}_{\mathrm{i}-1}, \mathrm{t}_{\mathrm{i}-1}\right)$

$=\left(\mathrm{x}_{\mathrm{i}}, \mathrm{t}_{\mathrm{i}}\right)$

As such, $\mathrm{h} \circ \phi=\psi \circ \mathrm{h}$. Therefore, the two flows are topologically conjugated.

\section{RESULTS}

In this study, we have shown that the flows of any two continuous-time dynamical systems with the same number of equilibrium points and trajectories, and has no periodic orbit can be conjugated topologically temporally. Besides, their trajectories are linearly ordered and order isomorphic to each other by the relation induced from their flow Fig. 1. By endowing the interval topology, both the LOTS are proven to be Hausdorff and homeomorphic to each other. 


\section{DISCUSSION}

In the theory of dynamical systems, classification problem is important as it provides a method to simplify objects under investigation and gives us an insight into the structure of dynamical system (Nguyen, 1996). The results obtained in this study are therefore crucial as it provides us another viewpoint in observing a dynamical system of our interests. One example can be found in (Tahir et al., 2010) whereby the dynamics of patient having epileptic seizure is showed transported to a visual platform namely, Flat EEG. Since they are topologically conjugate, they have the same topological properties (Robbin, 1972). Thus, we can study the topological properties of seizure from Flat EEG.

Epilepsy is a general term used for a group of disorders that cause disturbances in electrical signal of the brain. In epilepsy there is a miniature brainstorm of certain groups of brain cells and this is often associated with a sudden and involuntary contraction of a group of muscles and loss of consciousness. It can happen in a small area of the brain or the whole brain. Depending on the part of the brain that is affected, it causes involuntary changes in body movement or function, sensation, awareness, or behavior where these changes are known as epileptic seizure (Fig. 4a).

Electroencephalography (EEG) is the recording of electrical activity originating from the brain in contrast to Magnetoencephalography (MEG) which records the magnetic fields. It plays an important diagnostic role in epilepsy and provides supporting evidence of a seizure

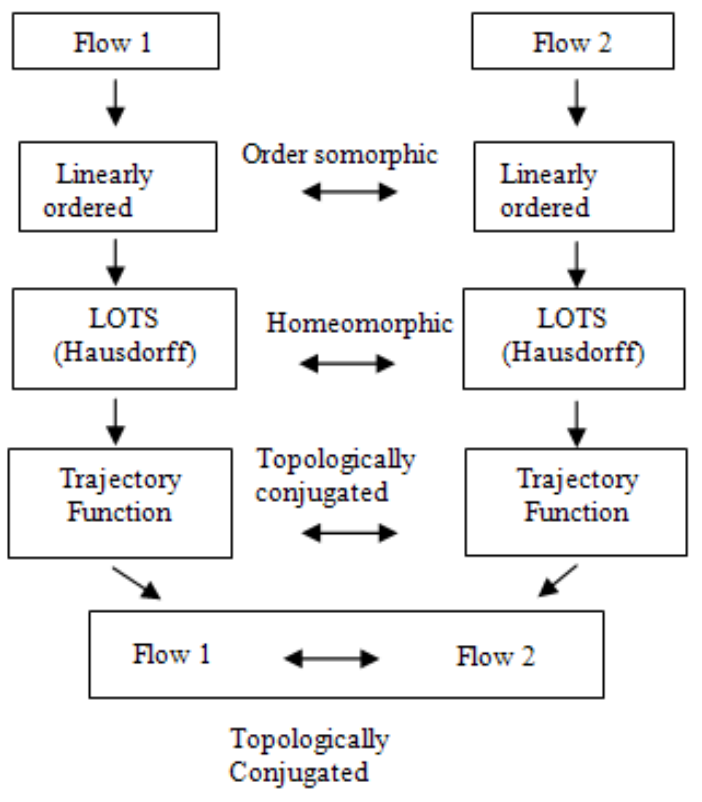

Fig. 1: Framework disorder as well as assisting neurologist in classifying seizures. EEG has been used extensively to record the abnormal brain activity associated with epileptic seizures (Fig. 2). It is recorded on the surface of the scalp using electrodes, thus the signal is retrievable non-invasively. The type of activity and the area of the brain that is recorded from EEG will assist the physician in prescribing the correct medication for certain type of epilepsy. Patients with epilepsy that cannot be controlled by medication will often have surgery in order to remove the damaged tissue. Thus the EEG plays an important role in localizing this tissue.

On the other hand, Flat EEG is a method for mapping high dimensional signal, namely EEG into a low dimensional space (MC) developed in (Tahir et al., 2006). The whole process of this novel model is consists of three main parts. The first part is flattening the EEG where the transformation of three dimensional space into two dimensional space that involved location of sensor in patients head with EEG signal (Fig. 3). This flattening process can preserves magnitude and orientation of the surface (Tahir et al., 2006). Secondly, the EEG is processed using Fuzzy C-Means (FCM) to obtain the number of cluster centers. Finally, the optimal number of clusters is determined using cluster validity analysis.

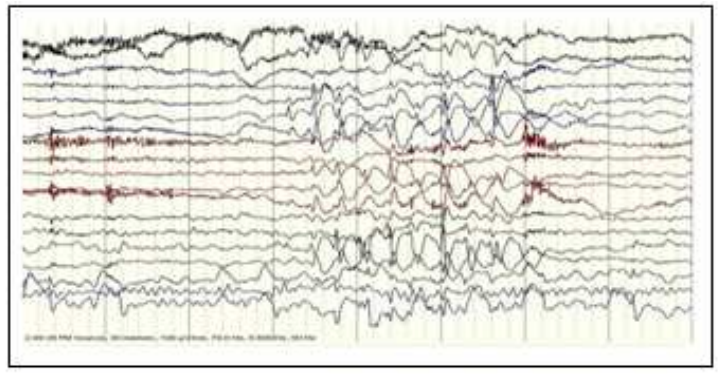

Fig. 2: EEG signal

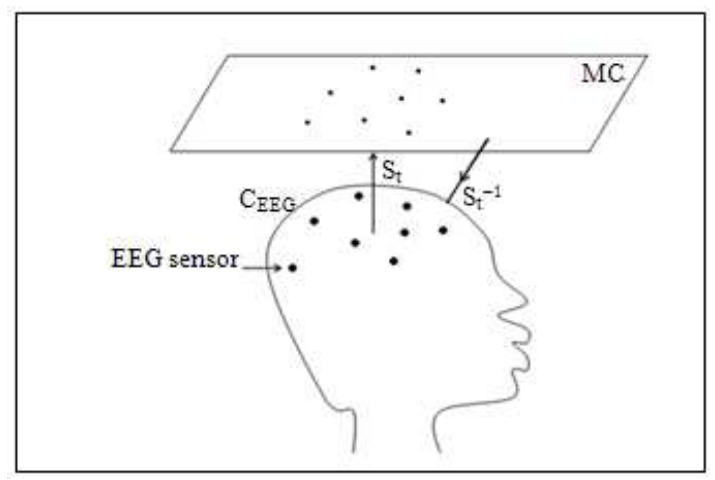

Fig. 3: EEG projection 


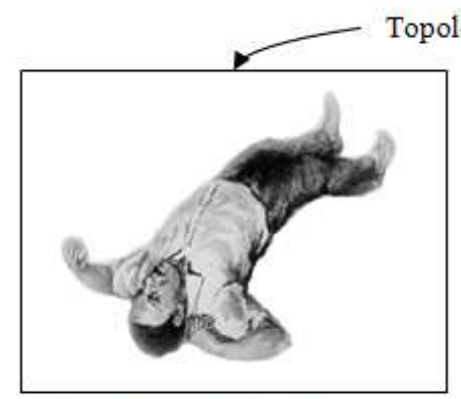

(a) Patient during seizure

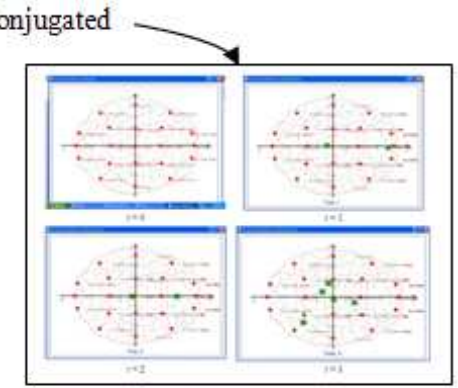

(b) Visual platform (Flat EEG)

Fig. 4: Topological conjugacy between seizure and Flat EEG

This new model enables tracking of brainstorm during seizure (Tahir et al., 2006). Figure 4b contains examples of Flat EEG. Red dots represent the electrodes while green dots represent the cluster centers after the transformation from the scalp of the patients. By using Theorem 6, the dynamics of epileptic seizure can be portrayed on Flat EEG (Fig. 4). For more details, see (Tahir et al., 2010).

\section{CONCLUSION}

In this study, the set of all continuous-time dynamical systems with the same number of equilibrium points and trajectories, and has no periodic orbit is showed to form an equivalence class under the relation of topologically conjugated. Firstly, two trajectories resulting from two distinct flows of continuous-time dynamical systems were composed into a set of points, and then it is showed that they exhibit linear ordering properties. Next, a function, $\theta$ is introduced to show the two set of points are order isomorphic by an induced relation and homeomorphic when endowed with the interval topology. The two trajectories are then showed conjugated topologically. By the arbitrariness in selection of flows and their respective initial states, we conclude that any two continuous-time dynamical systems with the same number of equilibrium points and trajectories, and have no periodic orbit share the same dynamics temporally. In addition to the results achieved, we present an application of it.

\section{ACKNOWLEDGEMENT}

The researchers would like to thank their family members for their continuous support and Ministry of Science, Technology and Innovation for granting the National Science Fellowship scholarship during his study. Authors also appreciate the financial support received from grant vot. 78397 .

\section{REFERENCES}

Adeqbie, K.S. and F.O. Alao, 2007. Flow of temperature-dependent viscous fluid between parallel heated walls: exact analytical in the solutions presence of viscous dissipation. J. Math. Stat., 3: 12-14. DOI: 10.3844/jmssp.2007.12.14

Artur, O. L., 1979. Stuctural stability and hyperbolic attractors. Trans. Am. Math. Soc., 252: 205-219. http://www.jstor.org/stable/1998085

Baryarama, F., L.S. Luboobi and J.Y.T. Mugisha, 2005. Periodicity of the HIV/AIDS epidemic in a mathematical model that incorporates complacency. Am. J. Infect. Dis., 1: 55-60. DOI: 10.3844/ajidsp.2005.55.60

Bennet, H.R. and D.J. Lutzer, 1996. Point-countability on generalized ordered spaces. Topol. Appli., 20: 1-17. DOI: 10.1016/0166-8641(96)00004-1

Bennet, H.R., R. Byerly and D.J. Lutzer, 2001. Cleavability in ordered spaces. Order, 18: 1-17. http://www.math.wm.edu/ lutzer/papers/BennetBy erlyLutzer.pdf

Chen, S.L., 2000. Cone of non-linear dynamical system and group preserving schemes. Pergamon. Inter. J. Non-Linear Mech., 36: 1047-1068. DOI: 10.1016/S0020-7462(00)00069-X

Eduard, G., L. Helwig and W. Raine, 1999. Visualization of dynamical systems. Elsevier. FGCS., 15: 75-86. DOI: 10.1016/S0167739X(98)00054-5

Erik, M. B. and D.S. Joseph, 2010. On Comparing Dynamical Systems by Defective Conjugacy: In: Symbolic Dynamics Interpretation of Commuter Functions, Physica, D. (Ed.). pp: 579-590. DOI: 10.1016/j.physd.2009.12.007

Eugene, M.I., 2007. Dynamical System in Neuroscience: In: The Geometry of Excitability and Bursting. 1st Edn., The MIT Press Cambridge, Massachusetts London England, ISBN: 978-0-26209043-8, pp: 8. 
Flytzanis, E., 1976. Linear dynamical systems. Proc. Am. Math. Soc., 55: 367-370. DOI: 10.1090/S0002-9939-1976-0407236-0

Ibrahim, G., M.A. Ramadan, S.A.A. El-Marouf and A.M. Al-Mahdi, 2007. Periodic and Non periodic (complex) behavior of a model of bioreactor with cell recycling. J. Math. Stat., 3: 1-11. DOI: 10.3844/jmssp.2007.1.11

James, D.M., 2007. Differential dynamical systems. Siam. Math. Model. Comp., 1: 105-108. ISBN: 0898716357, 9780898716351

Kopperman, R.D., E.H. Kronheimer and R.G. Wilson, 1998. Topologies on totally ordered sets. Topol. Appli., 90: 165-185. DOI: 10.1016/S01668641(97)00191-0

Krishan, M. M., L. Barazane and A. Khwaldeh, 2010. Using an adaptative fuzzy-logic system to optimize the performances and the reduction of chattering phenomenon in the control of induction motor. Am. J. Applied Sci., 7: 110-119. DOI: 10.3844/ajassp.2010.110.119

Ling, H. and N.M. Anthony, 2007. Unifying theory for stability of continuous, discontinuous and discretetime dynamical systems. Nonlinear Anal. Hybrid Syst., $\quad 1$ : 154-172. DOI: 10.1016/J.NAHS.2006.10.002

Mark, L.H. and D.L. Marc, 2005. The importance of dynamic systems approaches for understanding development. Dev. Rev., 25: 247-251. DOI: 10.1016/j.dr.2005.09.002

Nandhakumar, S., V. Selladurai and S. Sekar, 2009. Numerical investigation of an industrial robot arm control problem using haar wavelet series. Am. J. Eng. Applied Sci., 2: 584-589. DOI: 10.3844/ajeassp.2009.584.589
Nguyen, D.C., 1996. Topological classification of linear hyperbolic cocycles. J. Dynamics Differ. Equ., 8. DOI: 10.1007/BF02218762

Robbin, J.W., 1972. Topological conjugacy and structural stability for discrete dynamical systems. Bull. Am. Math. Soc., 78: 923-952. http://www.ams.org/journals/bull/1972-7806/S0002-9904-1972-13058-1/S0002-9904-197213058-1.pdf

Sekhar, V.C., L. Alexander, and M. H. Wassim, 1999. generalized lyapunov and invariant set theorems for nonlinear dynamical systems. Syst. Ctrl. Lett., 38: 289-295. http://soliton.ae.gatech.edu/people/whaddad/journa 1/1999generalized.pdf

Steve, R., 2008. Lattices and Ordered Sets. 1st Edn., Springer Science + Business Media, USA., pp: 13 . DOI: 10.1007/978-0-387-78901-9

Tahir, A., and L.K. Tan, 2010. Topological conjugacy between seizure and flat electroencephalography. Am. J. Applied Sci., 7: 1470-1476. DOI: 10.3844/ajassp.2010.1470.1476

Tahir, A., M.A. Jafri, Z. Fauziah, M. Faridah and A.M.H. Zabidi, 2006. Tracking the storm in the brain. J. Quantitative Meth., 2: 1-9.

Tahir, A., Z. Fauziah, J. Abdullah and F. Mustapha, 2005. Dynamical system of an epileptic seizure. Proceedings of the Asian Conference on Sensors and International Conference on New Techniques in Pharmaceutical and Biomedical Research, Sept. 6-7, IEEE Explore, Kuala Lumpur, pp: 78-80. DOI: 10.1109/ASENSE.2005.1564510

Tim, P. and P. Justin, 1989. Dynamical systems from function algebras. Proc. Am. Soc., 105: 80-86. http://www.jstor.org/stable/2046738 